\title{
Evolution-induced Catastrophe and its Predictability
}

\author{
Yu-Jie WeI, ${ }^{1}$ Meng-Fen Xia, ${ }^{1,2}$ Fu-Jiu Ke, ${ }^{1,3}$ XIAng-Chu Yin ${ }^{1,4}$ and \\ YI-LONG BAI ${ }^{1}$
}

\begin{abstract}
Both earthquake prediction and failure prediction of disordered brittle media are difficult and complicated problems and they might have something in common. In order to search for clues for earthquake prediction, the common features of failure in a simple nonlinear dynamical model resembling disordered brittle media are examined. It is found that the failure manifests evolutioninduced catastrophe (EIC), i.e., the abrupt transition from globally stable (GS) accumulation of damage to catastrophic failure. A distinct feature is the significant uncertainty of catastrophe, called sample-specificity. Consequently, it is impossible to make a deterministic prediction macroscopically. This is similar to the question of predictability of earthquakes. However, our model shows that strong stress fluctuations may be an immediate precursor of catastrophic failure statistically. This might provide clues for earthquake forecasting.
\end{abstract}

Key words: Evolution-induced catastrophe, sample-specificity, stress fluctuations, earthquake prediction, predictability.

\section{Introduction}

Earthquake prediction, the contemporary scientific challenge, is drawing more and more attention. This is mainly due to two factors: the importance of earthquake prediction and the difficulty of the problem.

Currently, it seems that efforts to identify deterministic precursors of earthquakes have been mostly unsuccessful. This arouses the discussion of whether an earthquake is predictable or not (GELLER et al., 1997). We still believe that earthquakes do have some incubating phase, although it seems still to be quite far beyond our knowledge. With many intriguing but fragmentary observations of possible precursory phenomena, people are frustrated again and again. Therefore,

\footnotetext{
${ }^{1}$ State Key Laboratory of Nonlinear Mechanics, Institute of Mechanics, Chinese Academy of Sciences, Beijing 100080, China. E-mail: weiyj@Inm.imech.ac.cn; E-mail: baiyl@lnm.imech.ac.cn

${ }^{2}$ Department of Physics, Peking University, Beijing 100871, China. E-mail: xiam@lnm.imech.ac.cn

${ }^{3}$ Department of Applied Physics, Beijing University of Aeronautics and Astronautics, Beijing 100083, China. E-mail: kefi@Inm.imech.ac.cn

${ }^{4}$ Center for Analysis and Prediction, China Seismological Bureau, Beijing 100036, China. E-mail: yinxc@btamail.net.cn
} 
perhaps we should seek alternative approaches to earthquake forecasting and seek sound bases for the approaches. If we assert that an earthquake is unpredictable now, we may throw the baby out with the bath water! (Bowman and SAMmis, 1999).

Actually, recent investigations have highlighted the key role of the heterogeneity of the crust and the nonlinear evolution of aggregations in the nucleation of earthquakes. The lithosphere of the solid earth can be regarded as a hierarchy of blocks with different length scales, from major tectonic plates to grains of rocks, which are interlinked by weak boundary zones or interfaces (TURCOTTE, 1992). "This implies that exact predictions are not possible. But it does not imply that earthquakes cannot be predicted (forecast) with considerable accuracy" (TURCOTTE, 1999). In fact, precursory clustering of earthquakes was investigated in terms of an aggregation model of crack growth and ultimate fusion on a multiplanar earthquake fault system with nonlinear rheology (YAMMASHITA and KNOPOFF, 1992). Other interesting attempts to understand earthquake forecasting in a nonlinear heterogeneous system include the stress release model (VERE-JONES, 1978), cellular automaton model (SornetTe and SAmmis, 1995; SAmmis and SMITH, 1999), particle-based lattice solid model (MORA and PlaCE, 1999), load/unload response ratio (YIN et al., 1995), and so on. Therefore, the complexity of earthquake prediction seems to be rooted in the nonlinear evolution of systems with disordered heterogeneity on multiple scales.

Aimed at such a class of heterogeneous materials whose failure is a nonlinear evolution, we construct a model based on brittle fracture. In comparison with earthquakes, there are two similar features (BAI et al., 1994a,b; XIA et al., 1996a,b, 1997; Ke et al., 1998):

1. Evolution-induced catastrophe (EIC). The failure usually exhibits an abrupt transition from globally stable accumulation of damage (GS) to catastrophic failure.

2. Sample-specificity. There may be a great diversity in catastrophe thresholds, i.e., catastrophic failure shows different behaviors sample-to-sample under identical macroscopic conditions. This leads to uncertainty of macroscopic failure.

In this paper a statistical analysis is performed for a simple nonlinear dynamical model of damage and failure, which demonstrates evolution-induced catastrophe and sample-specificity. Next an approach to distinguish the two phases of nonlinear evolution, namely, from GS to EIC is explored. The results show that the magnitude of fluctuations in the governing stress field appears to be a possible precursor of EIC. This may indicate that statistical warning signs in fluctuations before catastrophic failure may shed a light on earthquake forecasting.

The next section will introduce a nonlinear dynamical model of damage and failure called the coupled-pattern model. Thereafter, its evolution-induced catastro- 
phe and sample-specificity will be discussed. Finally, we try to explore precursors of the eventual failure.

\section{Coupled-pattern Model}

Consider a macroscopic system consisting of mesoscopic units. The system should be described by patterns mesoscopically. They are the pattern of material properties, the pattern of damage and the pattern of the governing stress field. The behavior of the system can be simulated by coupled-evolution of these patterns.

As an example, we consider a ring model (one-dimensional chains with periodic boundary condition) with $N$ units (XIA et al., 1994).

\section{(1) Patterns}

After denoting the strength of unit $i$ by $\sigma_{c i}$, the pattern of material properties is expressed by $\Sigma_{c}=\left\{\sigma_{c i}, i=1,2, \ldots, N\right\}$. In order to take heterogeneity into account, the values of $\sigma_{c i}$ are assumed to be random in the chain, but follow a distribution function $f\left(\sigma_{c}\right)$. We take the average strength $\bar{\sigma}_{c}=\int_{0}^{\infty} \sigma_{c} f\left(\sigma_{c}\right) d \sigma_{c}=1$. A sample is specified by its pattern $\Sigma_{c}$ mesoscopically. Then, samples with identical distribution functions $f\left(\sigma_{c}\right)$ are identical macroscopically.

The damage pattern is denoted by $X=\left\{x_{i}, i=1,2, \ldots, N\right\}$, where $x_{i}=0$ for an intact unit and $x_{i}=1$ for a broken unit. Macroscopically, the system is described by the damage fraction.

$$
p=\frac{1}{N} \sum_{i=1}^{N} x_{i}
$$

It is interesting to examine the damage process with increasing stress. The stress pattern $\Sigma=\left\{\sigma_{i}, i=1,2, \ldots, N\right\}$, where $\sigma_{i}$ is the ratio of the current stress on unit $i$ to $\bar{\sigma}_{c}$. Macroscopically, the governing parameter is the nominal stress $\sigma_{0}$ given by

$$
\sigma_{0}=\frac{1}{N_{i}} \sum_{i=1}^{N} \sigma_{i}
$$

There are two levels which describe damage evolution as shown in Table 1.

Table 1

Description for meso-macro levels

\begin{tabular}{llll}
\hline & Material property & Damage & Stress \\
\hline Meso-patterns & Strength pattern $\Sigma_{c}$ & Damage pattern $X$ & Stress pattern $\Sigma$ \\
Macro-parameters & Distribution of strength $f\left(\sigma_{c}\right)$ & Damage fraction $p$ & Nominal stress $\sigma_{0}$ \\
\hline
\end{tabular}




\section{(2) Load-sharing Rules}

The stress pattern $\Sigma$ is determined from the damage pattern $X$ according to load-sharing rules, which describe how the nominal stress of broken units is to be transferred to nearby intact units. Formally, the load-sharing rules can be expressed by "interactions" between intact units and broken units. For simplicity, we adopt a model in which the interaction is equal to the summation of intact-broken pair interactions. In this way, the stress on unit $i$ can be given by

$$
\sigma_{i}=\left(1-x_{i}\right)\left[1+\sum_{j \neq i} x_{i} a(|i-j|)\right] \sigma_{0}
$$

where $a(|i-j|)$ is the contribution of unit $j$ to unit $i$ and depends on the "distance" $|i-j|$. From equations (2) and (3), $a(|i-j|)$ satisfies

$$
p=\frac{1}{N} \sum_{i} \sum_{j}\left(1-x_{i}\right) x_{j} a(|i-j|)
$$

where only the contributions of terms with $i \neq j$ are included, because $\left(1-x_{i}\right) x_{j}=0$ as $i=j$.

In the following, we examine three types of the contribution function $a$, which roughly cover all possible stress redistributions.

Rule I: Global mean field

This is the simplest load-sharing rule,

$$
a=\frac{1}{N(1-p)} \text {. }
$$

Then,

$$
\sigma_{i}=\frac{\sigma_{0}}{(1-p)}
$$

The stress is uniformly shared by all intact units. This is a rule without stress fluctuations.

Rule II: Local interactions

$$
a(|i-j|)=\left\{\begin{array}{ll}
\text { const } & \text { for } \quad|i-j| \leq \Delta \\
0 & \text { otherwise }
\end{array} .\right.
$$

This rule depends on a fixed local scale $\Delta$. As $\Delta \rightarrow N$, the model approaches the global mean field model.

Rule III: Cluster load-sharing

This is a type of local mean field model with a cutoff due to broken clusters. A broken cluster consists of $l$ connecting broken units with intact units at its two 
sides. The nominal stress of a broken cluster is shared by its two neighboring intact clusters equally. A uniform stress distribution is assumed for each intact cluster. Thus, a unit in an $s$-intact cluster separating an $l$ - and an $r$-broken cluster supports a stress

$$
\sigma=\left(1+\frac{l+r}{2 s}\right) \sigma_{0}
$$

In this rule, local scale is determined by the local pattern of damage. Therefore, there may be multiple scales during the course of damage evolution. Then, the evolution shows a nonlocal feature essentially (without a characteristic scale).

Generally, for rules II and III, the stress pattern $\Sigma$ depends on the details of the damage pattern $X$, and there are stress fluctuations in the system. The stress fluctuations play an essential role in damage and failure.

\section{(3) Evolution Dynamics}

The evolution of damage in the system with disordered heterogeneity is very complicated. In order to investigate the universal behavior of such kind of nonlinear system, we introduce simplified dynamics as follows:

For a sample specified by a strength pattern $\Sigma_{c}=\left\{\sigma_{c i}, i=1,2, \ldots, N\right\}$, the evolution of the damage pattern $X=\left\{x_{i}, i=1,2, \ldots, N\right\}$ is governed by the stress pattern $\Sigma=\left\{\sigma_{i}, i=1,2, \ldots, N\right\}$ according to the dynamics:

$$
x_{i}(t+1)=x_{i}(t)+\Delta x_{i}(t)
$$

where

$$
\Delta x_{i}(t)=S\left(\sigma_{i}(t)-\sigma_{c i}\right), \quad i=1,2, \ldots, N
$$

and

$$
S(y)=\left\{\begin{array}{lll}
1, & \text { for } & y \geq 0 \\
0, & \text { for } & y<0
\end{array}\right.
$$

where $t$ is the ordinal number. At each step, as pattern $X$ changes, pattern $\Sigma$ must be redetermined from the new pattern $X$ according to the specified load-sharing rule. This is to say, the stress pattern $\Sigma$ and the damage pattern $X$ evolve in a coupled way. From equations (1) and (9), we can obtain a "time series" of the damage fraction $p(t)$.

For a sample with an initially intact pattern $X(0)=\left\{x_{i}(0)=0, i=1,2, \ldots, N\right\}$, as the nominal stress $\sigma_{0}$ increases from $\sigma_{0}=0$ slowly, the first damage event will occur at a value of $\sigma_{0}=\sigma_{0}^{(1)}$, depending on distribution function $f\left(\sigma_{c}\right)$. Keeping $\sigma_{0}=\sigma_{0}^{(1)}$, the system will evolve and approach an equilibrium pattern $X^{(1)}$ (with damage fraction $p^{(1)}$ ). As $\sigma_{0}$ increases, the above procedure repeats. Subsequently, 


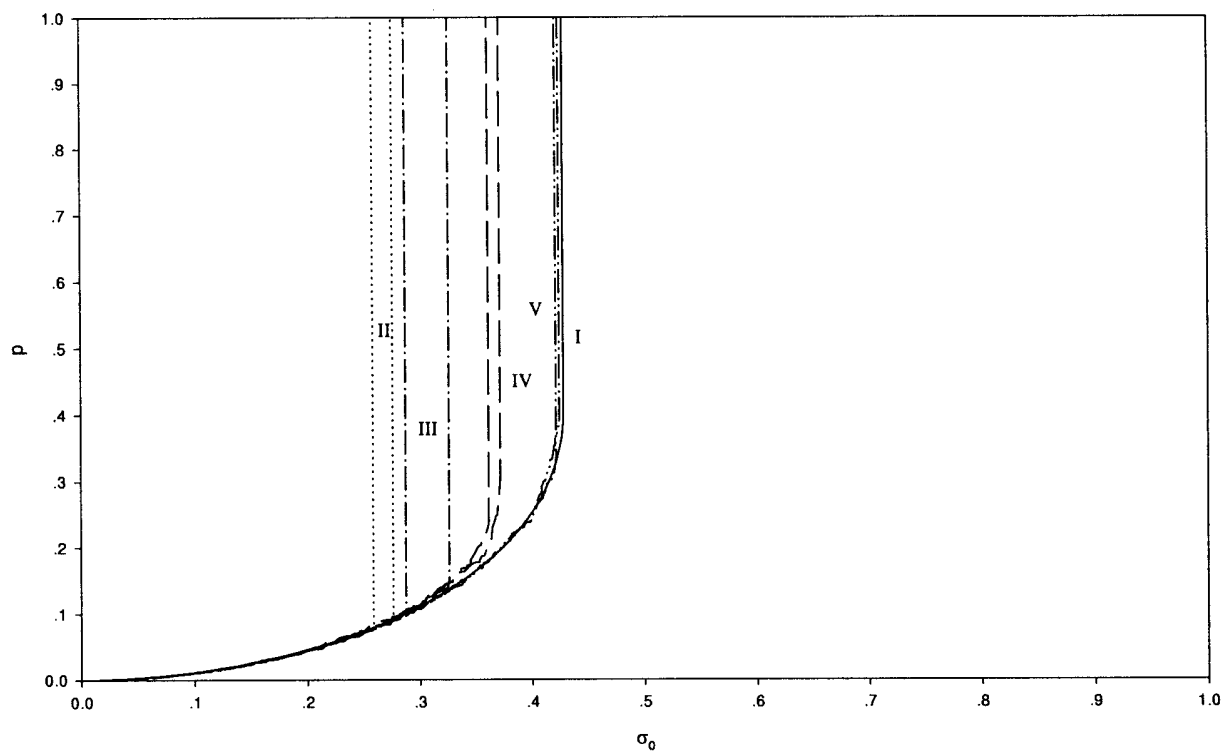

Figure 1

Equilibrium damage fraction $p$ as a function of imposed nominal stress $\sigma_{0}$ (with identical distribution functions $f\left(\sigma_{c}\right)$ ). I. Globally mean field; II. Cluster load-sharing rule, $N=20,000$; III. Cluster loadsharing rule, $N=2000$; IV. Local interaction, $\Delta=2, N=2000$; V. Local interaction, $\Delta=100, N=2000$.

we can obtain a series of stresses $\sigma_{0}^{(k)}$, equilibrium damage fractions $p^{(k)}$ and equilibrium damage patterns $X^{(k)}$. Eventually, failure will occur at $\sigma_{0}=\sigma_{0}^{\left(k_{f}\right)}=\sigma_{0 f}$.

\section{Evolution-induced Catastrophe and the Difficulty of Prediction}

Figure 1 shows the equilibrium damage fraction $p$ as a function of the imposed nominal stress $\sigma_{0}$, based on various load-sharing rules, as $\sigma_{0}$ increases quasi-statically. The strength distribution function $f\left(\sigma_{0}\right)$ adopted is a Weibull distribution

$$
f\left(\sigma_{c}\right)=m b \sigma_{c}^{m-1} \exp \left(-b \sigma_{c}^{m}\right)
$$

with $m=2$ and $b=1$ (then $\bar{\sigma}_{c}=1$ ). We also examined cases for various $f\left(\sigma_{c}\right)$. It is found that the system exhibits the following common behaviors.

\section{(1) Evolution-induced catastrophe}

The evolution of the system manifests two different modes: globally stable (GS) and evolution-induced catastrophic (EIC). There is a catastrophic threshold $\sigma_{0 f}$ for 
each sample. When $\sigma_{0}<\sigma_{0 f}$, the sample shows a stable accumulation of damage, called the globally stable mode (GS). This means that there are equilibrium patterns $X$ with damage fraction $p<1$ according to prescribed stresses $\sigma_{0}$. However, at $\sigma_{0}=\sigma_{0 f}$, the sample turns to catastrophic failure abruptly. This is to say, there are no equilibrium patterns with $p<1$ and the sample will go to failure $(p=1)$ unavoidably. This is called evolution-induced catastrophe (EIC). EIC is one of the fundamental modes of failure. $\sigma_{0 f}$ (and the relative damage fraction $p_{f}$ ) indicates the point of transition from the GS to the EIC mode.

\section{(2) Sample-specificity}

In the simulation, the samples with identical strength distribution function $f\left(\sigma_{c}\right)$ are regarded as identical samples macroscopically, however their mesoscopically disordered heterogeneity is specified by the strength pattern $\Sigma_{c}$. The failure behavior of a system following the global mean field rule is uniquely determined by function $f\left(\sigma_{c}\right)$. From equation (6), the equilibrium condition for specified $\sigma_{0}$ is given by equation $p=\int_{0}^{\sigma_{0} /(1-p)} f\left(\sigma_{c}\right) d \sigma_{c}$ and the failure threshold $\sigma_{0 f}$ can be deduced. That is to say, the macroscopically identical samples display identical behavior regardless of their mesoscopic details.

However, for samples with mesoscopic disorder and following load-sharing rules other than the global mean field rule, the macroscopic behavior, shows a clear difference sample-to-sample. Especially, there is a great diversity in catastrophic threshold $\sigma_{0 f}$ for the macroscopically identical samples. This distinct feature is called the sample-specificity of EIC. Sample-specificity results in uncertainty of material failure macroscopically.

\section{(3) Strength distribution}

The distribution function of the catastrophic threshold $W\left(\sigma_{0 f}\right)$ (shown in Fig. 2) can provide a probabilistic prediction of the catastrophe. For samples following the global mean field rule, there is a distinct failure strength $\sigma_{0 f}$ (Fig. 2). The macroscopic behaviors ( $p$ vs. $\sigma_{0}$ curves in Fig. 1) under other load-sharing rules deviate from that of the global mean field model. Catastrophe thresholds $\sigma_{0 f}$ for both global interaction and cluster load-sharing rules are usually considerably lower than that given by the global mean field model, and show a greater diversity (see Fig. 2).

Due to the sample-specificity of catastrophic failures, the macroscopic prediction of failure cannot be deterministic as in the global mean field model. Consequently, it is clear that the strengths of samples are statistically meaningful. This is helpful for material sciences, however not sufficient for rupture prediction. 


\section{Warning Signs of Catastrophe}

From the distinct differences in strength distributions, for the global mean field rule and the two other rules we are aware that the stress fluctuations play an essential role in evolution-induced catastrophe and sample-specificity. The main effects of stress fluctuations are as follows:

1. A significant reduction of catastrophe threshold from that derived from the global mean field model (Figs. 1 and 2).

2. The sample-specificity, especially the great diversity of catastrophe thresholds (Figs. 1 and 2).

The stress fluctuations can be measured by $\delta \sigma / \bar{\sigma}$, where

$$
\delta \sigma=\left[\frac{1}{N(1-p)} \sum_{i=1}^{N}\left(\sigma_{i}-\bar{\sigma}\right)^{2}\left(1-x_{i}\right)\right]^{1 / 2}
$$

is standard deviation of stress on intact units in the system, and

$$
\bar{\sigma}=\frac{\sigma_{0}}{1-p}
$$

is the mean stress of intact units.

Figure 3 delineates the stress fluctuations $\delta \sigma / \bar{\sigma}$ as a function of $p$ (the data are collected from the processes shown in Fig. 1). From Figure 3(a), we can see that, in the GS regime, the system remains in a state with a low level of stress fluctuations $(<1)$, nonetheless the fluctuations strengthen with increasing $p$. As the

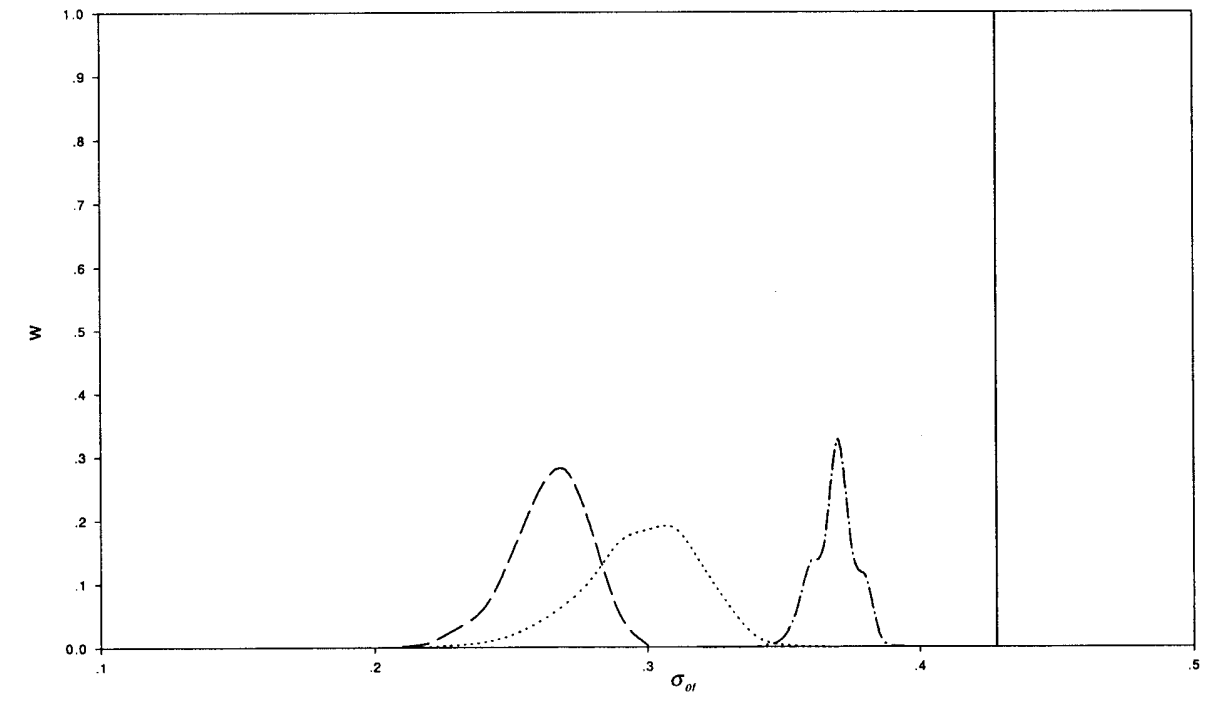

Figure 2

Statistical distribution function of the catastrophic threshold $W\left(\sigma_{f}\right)$. - Globally mean field; ---- Cluster load-sharing rule, $N=20,000 ; \cdots$ Cluster load-sharing rule, $N=2000$; ---- Local interaction, $\Delta=2$, $N=2000$. 
system turns to the EIC regime at $\sigma_{0}=\sigma_{0 f}\left(p=p_{f}\right)$, the stress fluctuations become considerably stronger than that in the GS regime. Figure $3(\mathrm{~b})$ shows similar behavior. For the local interaction model with sufficiently large $\Delta$ (see Fig. 3(c)), the behavior should approach that given by the global mean field model, and the stress fluctuations will remain at a low level.

Although it is impossible to find a deterministic criterion for catastrophic failure macroscopically due to sample-specificity, the above-mentioned results provide
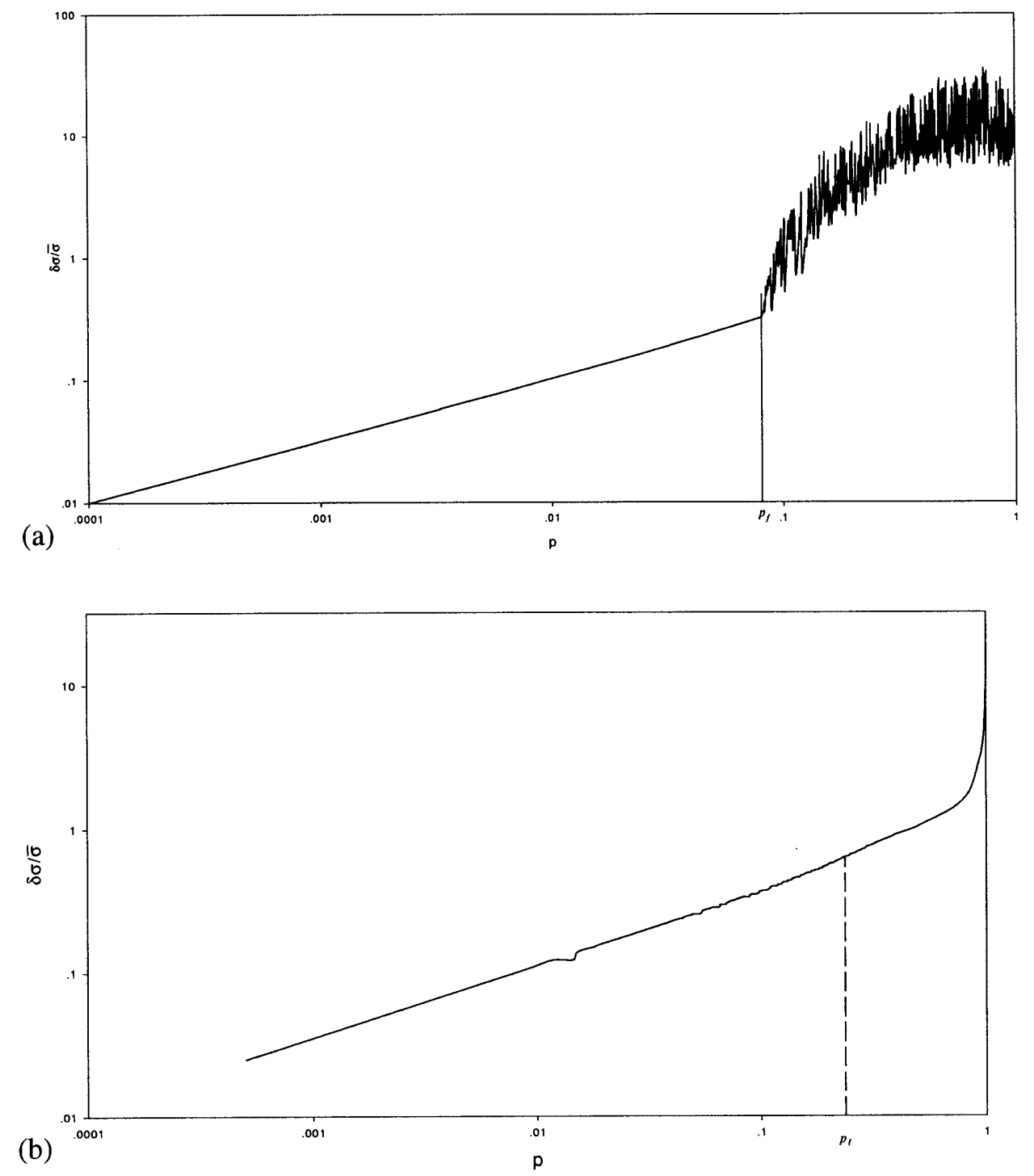
(c)

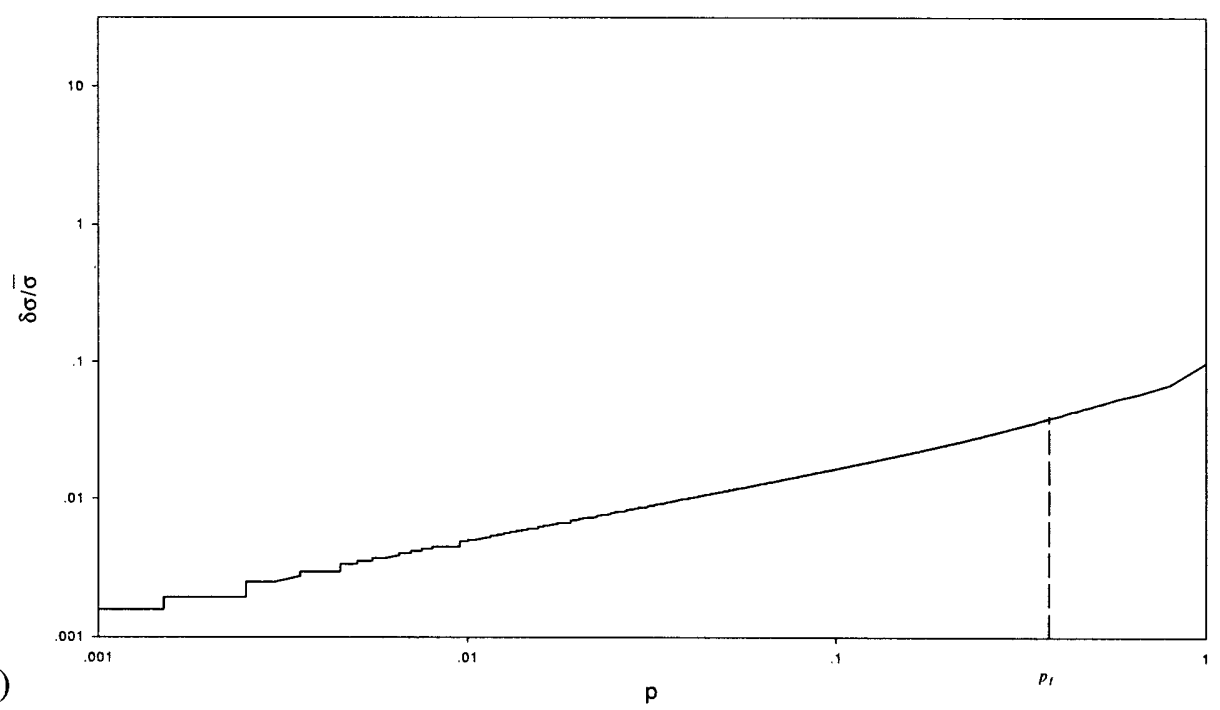

Figure 3

Stress fluctuation $\delta \sigma / \bar{\sigma}$ as a function of the damage fraction $p$. (a) Cluster load-sharing rule, $N=20,000$;

(b) Local interaction, $\Delta=2, N=2000$; (c) Local interaction, $\Delta=100, N=2000$.

clues to failure forecasting statistically. In this way we could possibly provide statistical warning signs based on an ensemble analysis. Let $\theta_{G S}$ be the maximum of stress fluctuations $\delta \sigma / \bar{\sigma}$ in the GS regime and $\theta_{E I C}$ be that in the EIC regime. The ensemble distributions $\Pi_{G S}(\theta)$ and $\Pi_{E I C}(\theta)$ are shown in Figure 4. From Figures 4(a), 4(b) and 4(c), we can see that the distribution functions $\Pi_{G S}(\theta)$ and $\Pi_{E I C}(\theta)$ are well separated from each other. Thus, statistically, we can set up a warning level of stress fluctuations to forecast the occurrence of catastrophic failure. For the local interaction model with sufficiently large $\Delta$ (say $\Delta=100$ for $N=2000$, see Fig. 4(d)), the distribution functions $\Pi_{G S}(\theta)$ and $\Pi_{E I C}(\theta)$ overlap each other. However, in this case, the behavior of the system is close to that of the global mean field model and failure prediction can be made from a deterministic criterion (see Fig. 1).

\section{Discussion}

The prediction of catastrophe is a problem of the first importance for engineering and natural hazards. However prediction is very difficult, especially for heterogeneous media like the crust. This is mainly due to the nonlinear evolution far from equilibrium and the catastrophic failure exhibited as a cascade from smaller scales to larger ones. Even in the EIC regime, there may be no significant signs in the initial stage of the catastrophic mode. The characteristics of catastrophe become visible only after it is enhanced strongly during nonlinear evolution. Secondly, due 
to sample-specificity, there is no deterministic, macroscopic criterion for catastrophe. However, for a system with disordered heterogeneity on multiple scales, it is usually impossible to secure a detailed description mesoscopically. Furthermore, sample-specificity implies that there is a sensitive link between macroscopic and mesoscopic scales. In other words, the macroscopic behaviors of a system may be sensitive to subtle details on mesoscopic scales. Consequently, an approach to failure prediction based upon statistical averages may be insufficient and may even be inappropriate.

In order to overcome the difficulty resulting from sample-specificity, we must find some delicate features which can be adopted to distinguish the GS and EIC

(a)

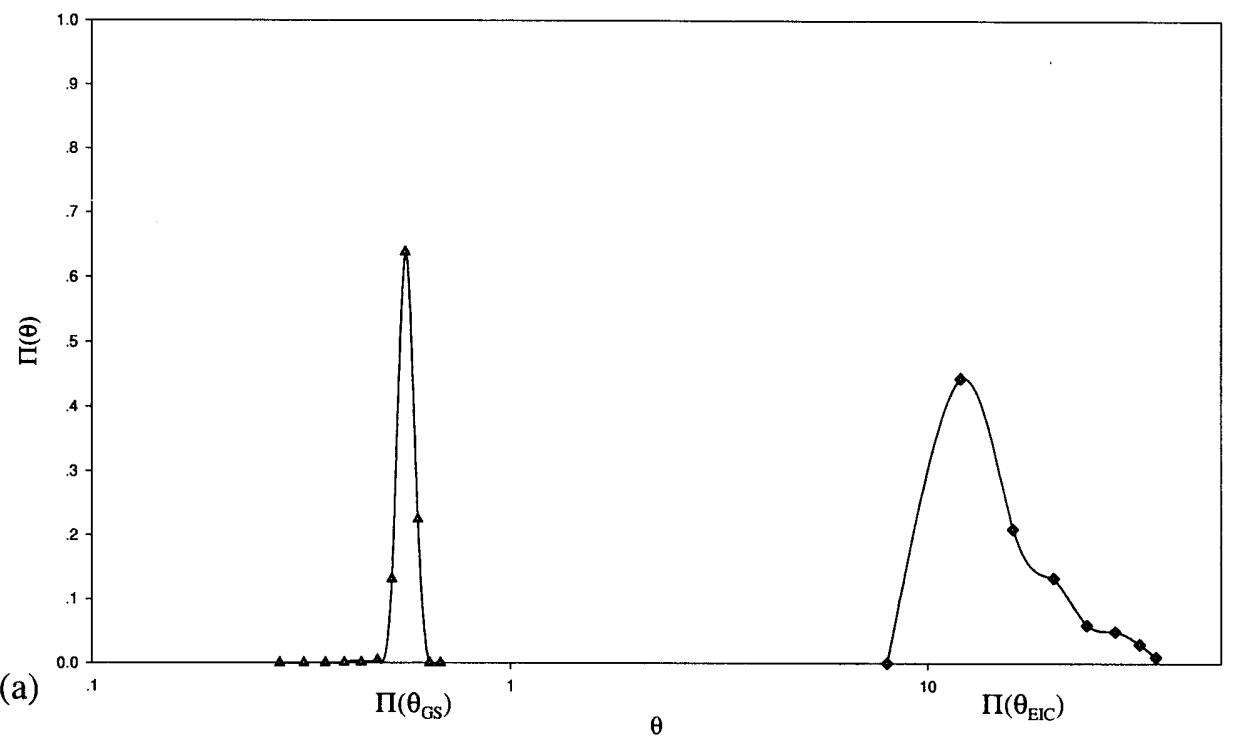

(b)

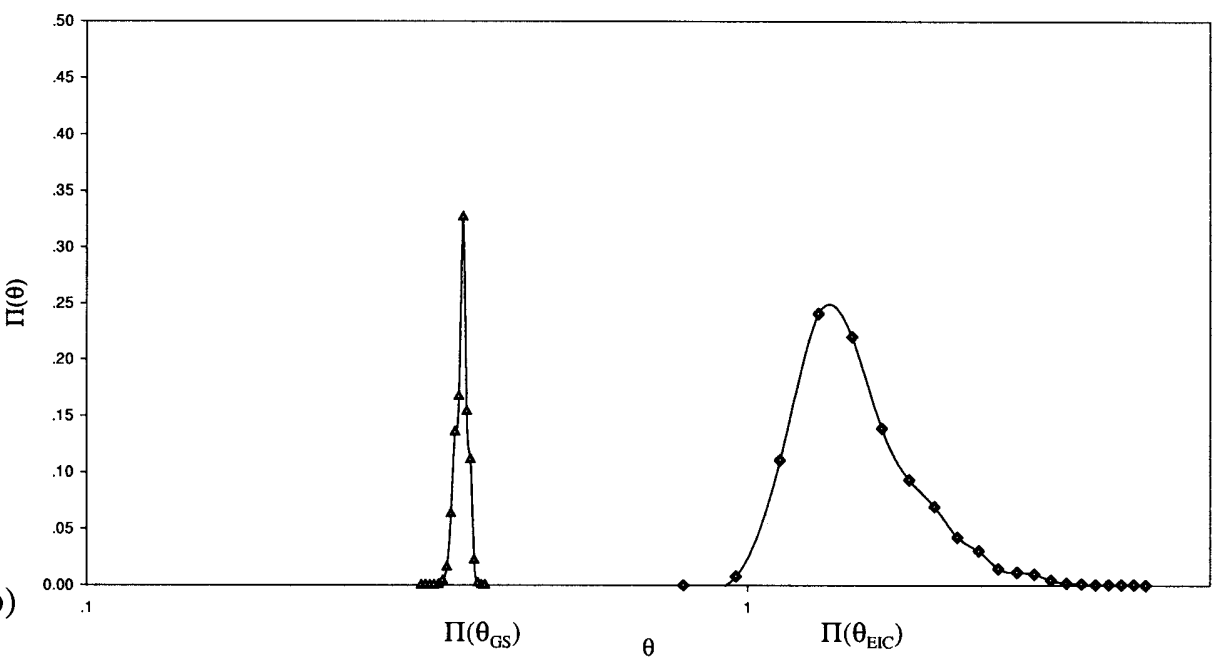


(c)
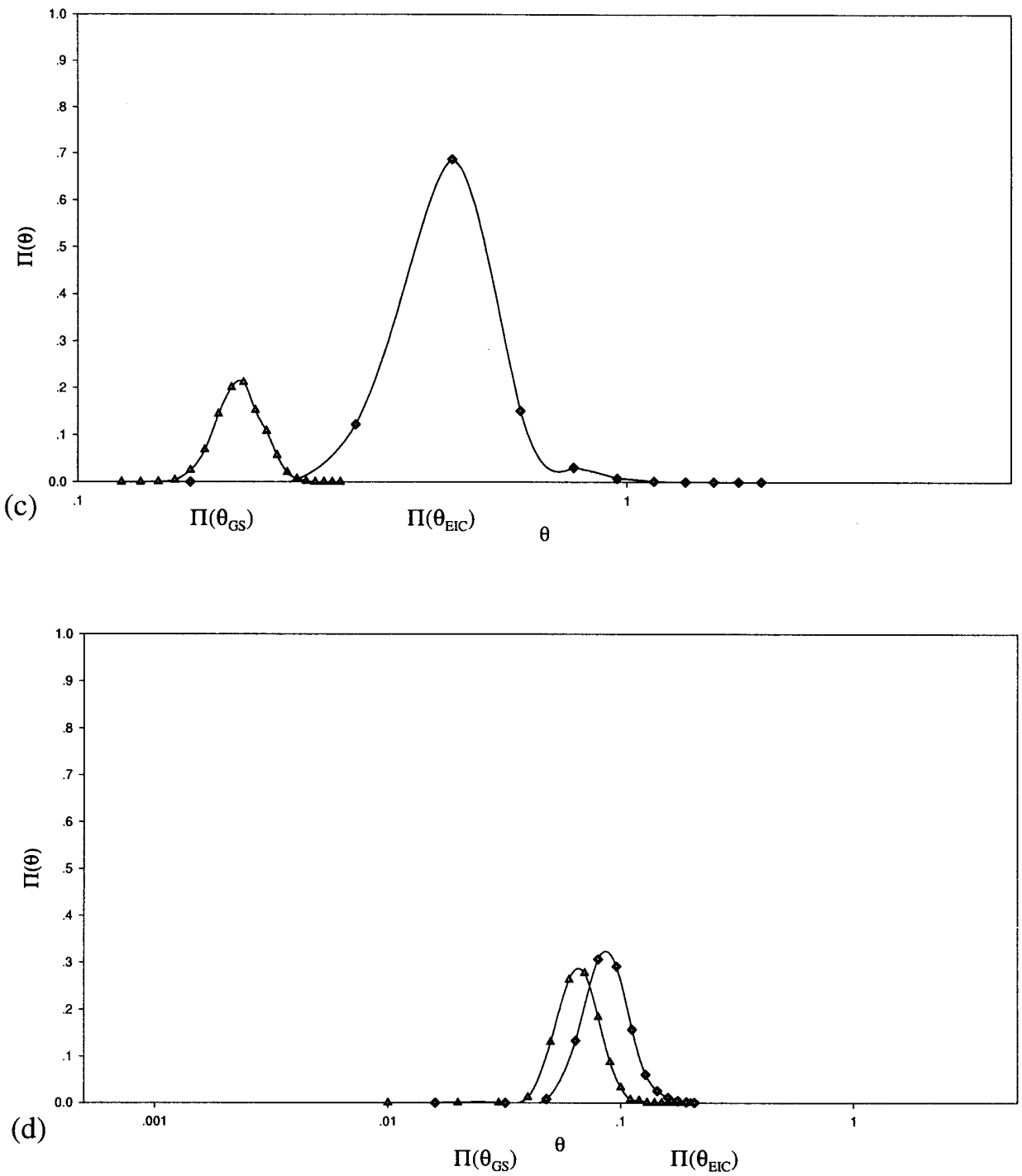

Figure 4

Statistical distribution function of maximum stress fluctuation $\theta_{G S}$ and $\theta_{E I C}$ in the GS and EIC regimes, respectively. $\Delta: \Pi\left(\theta_{G S}\right) \diamond: \Pi\left(\theta_{E I C}\right)$. (a) Cluster load-sharing rule, $N=20,000$; (b) Local interaction, $\Delta=2, N=2000$; (c) Local interaction, $\Delta=10, N=2000$; (d) Local interaction, $\Delta=100, N=2000$.

modes. Statistically, monitoring the fluctuations of the governing stress field in a sample may be a possible approach as discussed in the previous section. The important feature is that the fluctuations of the governing stress field will statistically be enhanced to a higher level as the evolution mode transits from the GS to EIC. Therefore, as the fluctuations of the governing field go beyond a warning level, we 
could note that the evolution of the system is entering EIC mode and catastrophic failure may occur. This approach might provide clues for earthquake prediction.

\section{Acknowledgements}

This work is supported by the National Fundamental Research Project "Nonlinear Science" and National Natural Science Foundation of China (No. 19732060, 19972004, 19572072 and 19577102). Computation was supported by the State Key Laboratory of Scientific and Engineering Computing.

\section{REFERENCES}

BAI, Y. L., KE, F. J., and XIA, M. F. (1994a), Deterministically Stochastic Behavior and Sensitivity to Initial Configuration in Damage Fracture, Science Bulletin (in Chinese) 39, 892-895.

BAI, Y. L., LU, C. S., KE, F. J., and XIA, M. F. (1994b), Evolution-induced Catastrophe, Phys. Lett. A $185,196-200$.

Bowman, D., and Sammis, Ch. (1999), An E-mail contribution for the debate on web site www.nature.com/ debates/earthquake/, A case for intermediate-term earthquake prediction: Don't throw the baby out with the bath water!

Geller, R. J., Jackson, D. D., Kagan, Y. Y., and Mulargia, F. (1997), Earthquakes Cannot be Predicted, Science $275,1616-1617$.

Ke, F. J., FAnG, X., XIA, M. F., ZhaO, D. W., and BAI, Y. L. (1998), Contingent Sensitivity to Configuration in a Nonlinear Evolution System, Prog. in Nat. Sci. 8, 170-173.

Mora, P., and Place, D., Accelerating energy release prior to large events in simulated earthquake cycles: Implications for earthquake forecasting. In Proc. ACES Inaugural Workshop, 31 Jan.-5 Feb., 1999, Brisbane and Noosa, QLD, Australia, pp. 195-200.

Sornette, D., and SAmmis, C. G. (1995), Complex Critical Exponents from Renormalization Group Theory of Earthquakes: Implications for Earthquake Predictions, J. Phys. 15, 607-619.

Sammis, C. G., and Smith, S. (1999), Seismic Cycles and the Evolution of Stress Correlation in Cellular Automaton Models of Finite Fault Networks, Pure appl. geophys. 155, 307-334.

Turcotte, D. L., Fractals and Chaos in Geology and Geophysics (Cambridge University Press, Cambridge 1992).

TURCOTTE, D. L., The physics of earthquakes: Is it a statistical problem? In Proc. ACES Inaugural Workshop, 31 Jan.-5 Feb., 1999. Brisbane and Noosa, QLD, Australia, pp. 95-98.

Yammashita, T., and KnOPOFF, L. (1992), Model for Intermediate-term Precursory Clustering of Earthquakes, J. Geophys. Res. 97 (B13), 19,873-19,879.

Vere-Jones, D. (1978), Earthquake Prediction: A Statistician's View, J. Phys. Earth 26, 129-146.

Xiang-Chu Yin, Xue-Zhong Chen, Zhi-Ping Song, and Can Yiu (1995), A New Approach to Earthquake Prediction - The Load/Unload Response Ratio (LURR) Theory, Pure appl. geophys. 145 (3/4), 701-715.

XIA, M. F., BAI, Y. L., and KE, F. J. (1994), Statistical Description of Pattern Evolution in Damage-fracture, Science in China (A) 37, 331-340.

XIA, M. F., Ke, F. J., BAI, J., and BAI, Y. L. (1997), Threshold Diversity and Trans-scales Sensitivity in a Nonlinear Evolution Model, Phys. Lett. A 236, 60-64.

XIA, M. F., Song, Z. Q., Xu, J. B., ZhaO, K. H., and BAI, Y. L. (1996a), Sample-specific Behavior in Failure Models of Disordered Media, Communication in Theoret. Phys. 25, 49-54.

XIA, M. F., BAI, Y. L., and Ke, F. J. (1996b), A Stochastic Jump and Deterministic Dynamics Model of Impact Failure Evolution with Rate Effect, Theoret. Appl. Fract. Mech. 24, 188-196.

(Received July 22, 1999, revised February 1, 2000, accepted April 15, 2000) 